\title{
An Adjustment of the Apparent Luminosity of Standard Candles for the 'De-boosting' Effect
}

\author{
Mark Zilberman \\ Shiny World Corporation, Ontario, Canada
}

\begin{abstract}
"De-boosting" is a well-known relativistic effect that alters the apparent luminosity of radiation sources with the non-zero redshift parameter z. It exists in both Special Relativity and General Relativity frameworks and is proportional to $(z+1)^{-2}$. While the "boosting" (for blueshift) and "de-boosting" (for redshift) of light sources has been successfully accounted for and observed in research of various astronomical objects, it was ignored in the establishment of Standard candles for cosmological distances. A Standard candle adjustment appears necessary for "de-boosting" for high z, otherwise we would incorrectly assume that Standard candles appear dimmer, not because of "de-boosting" but because of the excessive distance, which would affect the entire Standard candles ladder at cosmological distances. The "de-boosting" correction of the apparent luminosities of SNIa places the effective rest-frame magnitudes below the curve corresponding to the cosmological model with the parameters $\Lambda=0$ and $\left(\Omega_{\mathrm{M}}, \Omega_{\Lambda}\right)=(2,0)$ on the Hubble diagram. This way, the "de-boosting" correction of the apparent luminosities of SNIa may further adjust and clarify cosmological models.
\end{abstract}

Key words: galaxies: distances and redshifts - distance scale

\section{Introduction}

"Doppler boosting" is a well-known Special Relativity (SR) effect that increases the apparent luminosity of approaching light sources. Specifically, it allows to obtain the intrinsic value of the luminosities $\left(\mathrm{L}_{\mathrm{o}}\right)$ of relativistic objects by their apparent luminosity $(\mathrm{L})$, velocity and spectral index. "Doppler de-boosting" (Zhou \& Su 2006; Yang 2010) is the term used for the same relativistic effect calculated and observed for receding sources of radiation.

Relationship $\mathcal{M}(\mathrm{z}, \alpha)$ between the apparent $(\mathrm{L})$ and intrinsic luminosities $\left(\mathrm{L}_{\mathrm{o}}\right)$ as a function of redshift (z) and spectral index $(\alpha)$ was derived within the SR framework by (Zilberman 2021) from the (Lister 2003) formula, as

$\mathcal{M}(\mathrm{z}, \alpha) \equiv \mathrm{L} / \mathrm{L}_{\mathrm{o}}=(\mathrm{z}+1)^{\alpha-3}$

Since for type Ia supernova (SNIa), which are considered to be Standard candles on cosmological distances, per (Deng Wang \& Xin-He Meng, 2018) the spectral index $\alpha$ is approximately 1 , the

$\mathcal{M}(\mathrm{z}, \alpha) \equiv \mathrm{L} / \mathrm{L}_{0}=(\mathrm{z}+1)^{-2}$

The General Relativity (GR) framework produces gravitational de-boosting with the

same ratio $(\mathrm{z}+1)^{-2}$ under quite general assumptions, namely that photons travel along null geodesics of the spacetime metric and that the number of photons is conserved (Etherington, 1933, Ellis et al., 2012, Boero \& Moreschi, 2018).

"Boosting" and "de-boosting" were successfully taken into account in the analysis of relativistic jets of active galactic nuclei (AGN), in observations of double white dwarfs, in research of pulsars, in search of exoplanets and stars in binary systems, and in the analysis of 
gamma-ray bursts (GRBs) (Kellermann, Kovalev \& Lister 2007; Lister 2003; Shporer et al., 2010; Li K. L. et al, 2018; Placek 2019; Massi \& Torricelli-Ciamponi 2014).

Within cosmology however, the "de-boosting" effect was not accounted for in the establishment of Standard candles for cosmological distances. For distant galaxies we understandably cannot observe "boosting" because of redshift $\mathrm{z}>0$ due to the expansion of space and peculiar velocities of SNIa within parent galaxies are negligible compared to z. However, we have no reason to ignore the "de-boosting" effect, which is produced by the expansion of the universe or to assume that Type Ia supernovae are exceptions to this effect.

\section{Analysis}

Following (Ellis et al., 2012), in physical terms the first factor $(1+\mathrm{z})^{-1}$ in the GR de-boosting formula is the result of time dilation. We receive less photons per unit of time than were emitted. The second factor $(1+\mathrm{z})^{-1}$ is the drop of energy produced by redshift.

As such, for the analysis of de-boosting, the source of redshift $\mathrm{z}$ (speed in SR or gravity in GR) is not actually important. As soon as red shift $\mathrm{z}$ occurs due to time dilation, the de-boosting of the apparent luminosity also occurs in both SR and GR and is proportional to $(z+1)^{-2}$. For astronomical observations it implies a correction for de-boosting in the analysis of objects with redshift $\mathrm{z}$ when we calculate the luminosity distance.

The peculiar velocity of SNIa within parent galaxies may also add boosting/de-boosting input to apparent luminosity. However, we will not take this into account in this article since $\mathrm{z}_{\mathrm{p}}$ produced by the peculiar velocities of stars are negligibly less than the $\mathrm{z}$ of parent galaxies produced by the expansion of the universe.

In accordance to (Granot \& Ramirez-Ruiz, 2012) "for a relativistic source moving with a Lorentz factor $\Gamma=\left(1-\beta^{2}\right)^{-1 / 2} \gg 1$ (in the lab frame), half of the photons and most (3/4) of the emitted energy are within an angle of $1 / \Gamma$ around its direction of motion".

The illustration fig.1 (re-drawn from figure 11.2 of Granot \& Ramirez-Ruiz, 2012) illustrates the distribution of energy in "boosting" and "de-boosting" zones of relativistic radiation sources. The arrows show the direction of photons in the observer's frame for a source that emits isotropically in its own rest frame and moves to the right at different velocities. For supernovae located on cosmological distances we are always located in the "de-boosting" zone.

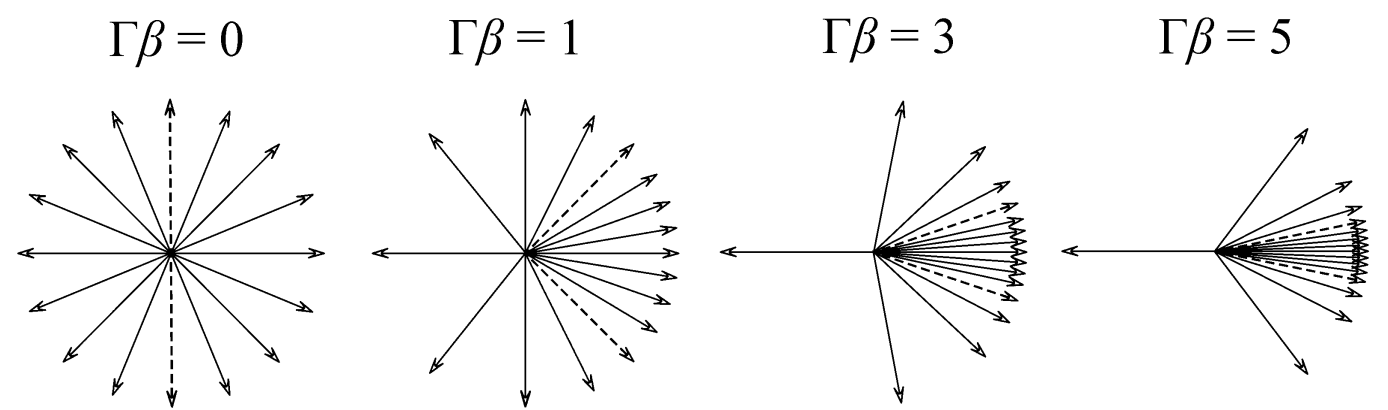

Figure 1. Relativistic boosting and de-boosting of a source that emits isotropically in its own rest frame and moves to the right at different velocities. Half of the photons (and 3/4 of the radiated energy) are within an angle of $1 / \Gamma$ around the direction of motion (between the dashed arrows, which correspond to $\theta=90^{\circ}$ in rest frame.) Re-drawn from Granot \& Ramirez-Ruiz, 2012. 
Although disregarding "de-boosting" for low-speed Standard candles simplifies calculations, for Standard candles with $\mathrm{z}>0.1$ the correction of "de-boosting" appears to be necessary. Otherwise, we would incorrectly assume that Standard candles appear dimmer, not because of "de-boosting," but because they are located further away than they truly are. This would affect the entirety of the Standard candles ladder at cosmological distances and cosmological models.

Per (Lister 2003), the relationship between the apparent luminosities (L), intrinsic luminosities $\left(\mathrm{L}_{0}\right)$ and spectral index is described by the following formulas:

$\mathrm{L}=\mathrm{L}_{\mathrm{o}} \delta^{\mathrm{p}}$

where the Doppler factor $\delta$ is

$\delta=\gamma^{-1}(1-\beta \cos \theta)^{-1}$

the Lorentz factor $\gamma$ is

$\gamma=\left(1-\beta^{2}\right)^{-1 / 2}$

the velocity $\beta$ is the speed $v$ of a relativistic light source normalized to the speed of light $c$

$\beta=v / c$

$\theta$ is the angle between line of sight and the velocity direction, $\alpha$ is the spectral index $\left(S_{v} \propto v^{\alpha}\right)$, and $\mathrm{p}=3-\alpha$ for a discrete emitting region.

"Doppler de-boosting" (Zhou \& Su 2006; Yang 2010) is the term of the same relativistic effect calculated and observed for receding sources of radiation. While "Doppler boosting" alters the apparent luminosity of approaching $\left(0<=\theta<=90^{\circ}\right)$ sources to be greater, "Doppler deboosting" alters the apparent luminosity of receding $\left(90^{\circ}<\theta<=180^{\circ}\right)$ sources to be fainter.

The following presents "Doppler de-boosting" using the redshift parameter z. As for cosmological objects the angle $\theta=180^{\circ}$, the Doppler factor $\delta$ is shown as

$\delta=\gamma^{-1}(1+\beta)^{-1} \equiv\left(1-v^{2} / \mathrm{c}^{2}\right)^{1 / 2}(1+v / \mathrm{c})^{-1}$

and since the redshift parameter $\mathrm{z}$ and velocity $v$ are related as

$\mathrm{z}+1=(1+v / \mathrm{c})\left(1-v^{2} / \mathrm{c}^{2}\right)^{-1 / 2}$

the $\delta$ and $\mathrm{z}$ are related as

$\delta=1 /(\mathrm{z}+1)$

As such, for the discrete emitting object parameter $p=3-\alpha$ (Lister 2003), the apparent luminosities $(\mathrm{L})$, redshift parameter $\mathrm{z}$, and the intrinsic luminosities $\left(\mathrm{L}_{\mathrm{o}}\right)$ relation is shown as

$\mathrm{L}=\mathrm{L}_{\mathrm{o}} /(\mathrm{z}+1)^{3-\alpha} \equiv \mathrm{L}_{\mathrm{o}}(\mathrm{z}+1)^{\alpha-3}$

or $\mathcal{M}(\mathrm{z}, \alpha) \equiv \mathrm{L} / \mathrm{L}_{\mathrm{o}}=(\mathrm{z}+1)^{\alpha-3}$

where $\mathcal{M}(\mathrm{z}, \alpha)$ is the ratio between the apparent and intrinsic luminosities as a function of redshift $\mathrm{z}$ and spectral index $\alpha$.

The general relativity framework produces an identical result under quite general assumptions, namely that photons travel along null geodesics of the spacetime metric and that the number of photons is conserved (Ellis et al., 2012, Boero \& Moreschi, 2018). The sole 
difference between formula (11) and (7.35) in Ellis et al., 2012 is the coefficient related to the spectral index correction, denoted in Ellis et al., 2012 as "C".

Regarding the spectral index $\alpha$ in formula (11). For Type Ia supernova (SNIa), which are considered to be Standard candles on cosmological distances, per (Deng Wang \& Xin-He Meng, 2018) the spectral index $\alpha$ is approximately 1 (per the "Joint Light-curve Analysis" sample containing 740 SNIa data points) and for the supernova remnant RCW 86 the spectral index is between 1.5 and 2 (Abramowski at al., 2018). In other words, for SNIa we can expect a relationship between $\mathrm{L}$ and $\mathrm{L}_{\mathrm{o}}$ as

$\mathcal{M}(\mathrm{z}) \equiv \mathrm{L} / \mathrm{L}_{\mathrm{o}}=(\mathrm{z}+1)^{-2}$

in both SR and GR.

Chart fig. 2 represents the influence of de-boosting to the apparent luminosities of SNIa for spectral indices $\alpha=1$ and $\alpha=2$, i.e. $\mathcal{M}(\mathrm{z})=(\mathrm{z}+1)^{-2}$ and $\mathcal{M}(\mathrm{z})=(\mathrm{z}+1)^{-1}$.

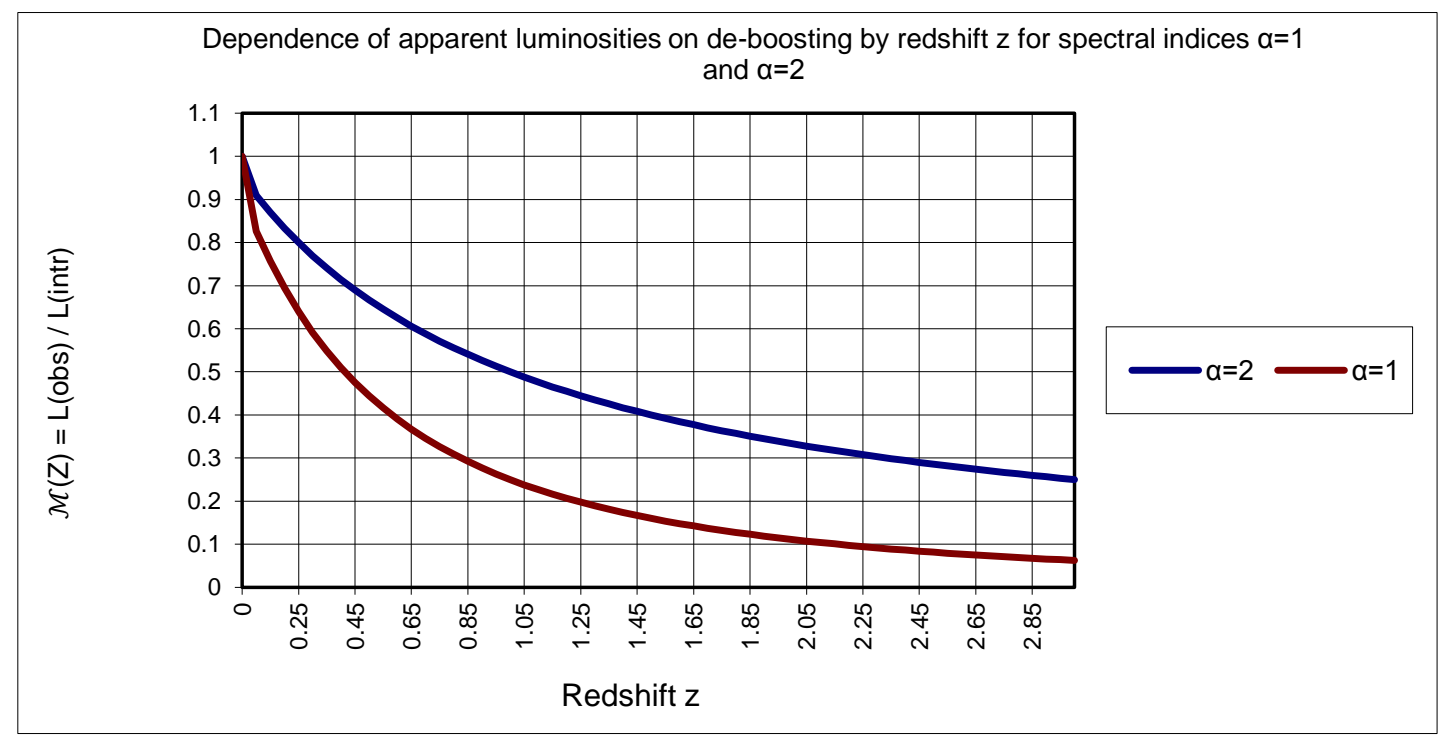

Figure 2. Influence of de-boosting to the apparent luminosities of SNIa for spectral indexes $\alpha=1$ (red line) and $\alpha=2$ (blue line).

Therefore, the luminosity of objects receding from the observer with a redshift of $\mathrm{z}=3$ appears 4 times fainter for spectral index $\alpha=2$ and 16 times fainter for spectral index $\alpha=1$. If we do not consider the "de-boosting" effect, we can incorrectly assume that these objects are located many times further away than they truly are.

Converting the de-boosting formula (12) into magnitudes we have $\Delta \mathrm{M}=-2.5 \log _{10} \mathcal{M}(\mathrm{z})=-2.5 \log _{10}\left[(\mathrm{z}+1)^{-2}\right]$

Chart fig. 3 presents the influence of de-boosting to the apparent luminosities of SNIa presented in magnitudes. 


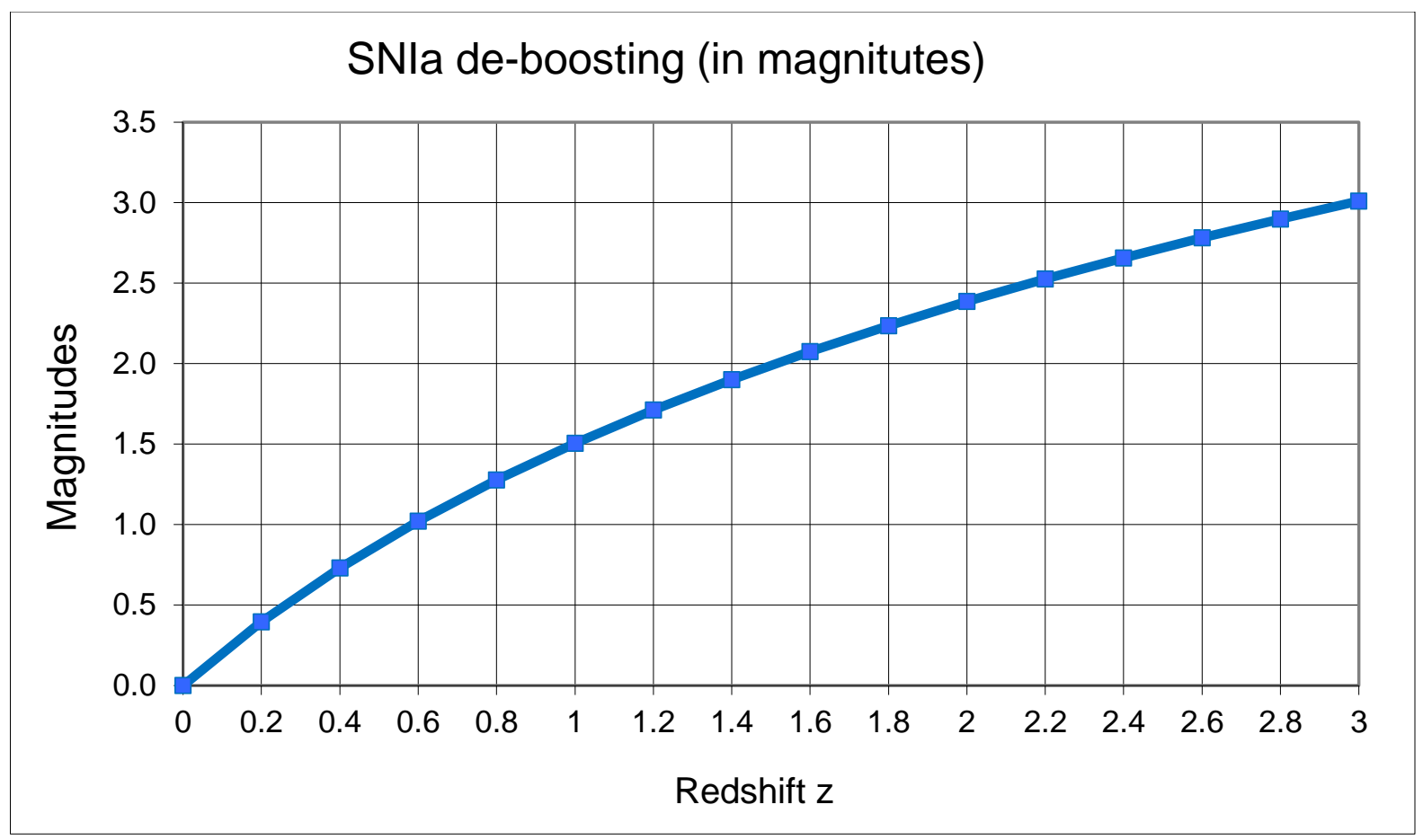

Figure 3. Influence of de-boosting to the apparent luminosities of SNIa in magnitudes.

\section{Accounting for the "de-boosting" effect in cosmology}

Using formula (13) we can check if accounting for "de-boosting" influences the cosmological models build on observation of SNIa as standard candles. To answer this question, we used data for SNIa published by (Perlmutter et al, 1999). Columns (2) and (3) in table 1 contain the redshift values $\mathrm{z}$ and effective rest-frame B magnitudes copied from columns (2) and (9) of Tables 1 and 2 in Perlmutter et al, 1999.

Column (4) of table 1 presents the corrections (in magnitudes) for de-boosting, derived from (13) as

Correction $=\Delta \mathrm{M}=2.5 * \log _{10}\left[(\mathrm{z}+1)^{-2}\right]$

Column (5) contains the corrected magnitudes as column (5) = column (3) + column (4).

Values from column (5) are presented in Fig. 4 below. The blue dots correspond to the magnitudes from the original publication (Perlmutter et al, 1999). The pink dots correspond to the magnitudes corrected for de-boosting.

The solid curve presenting the cosmological model with parameter $\Lambda=0$ and $\left(\Omega_{\mathrm{M}}, \Omega_{\Lambda}\right)=(2,0)$ was redrawn from (Perlmutter et al, 1999). 


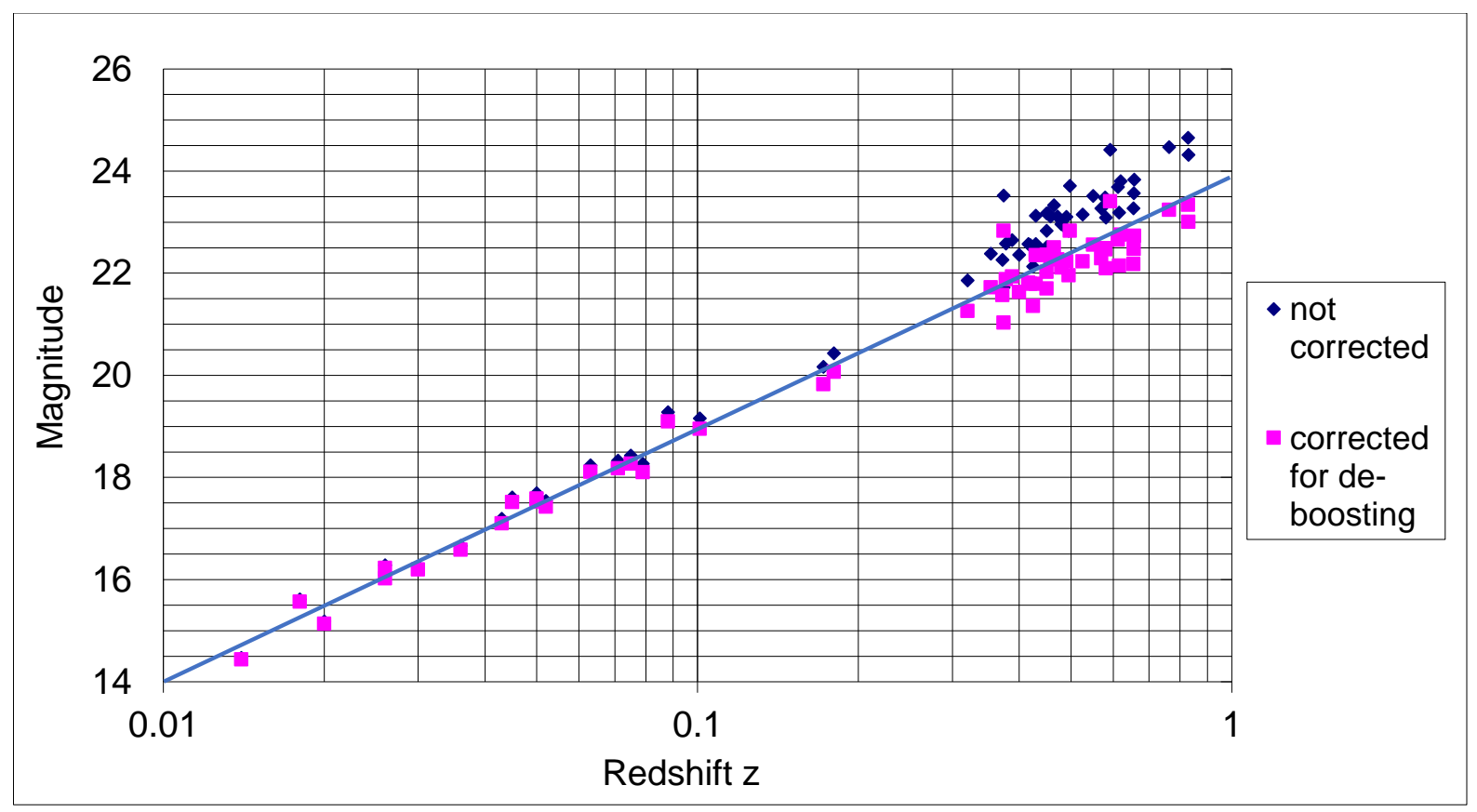

Figure 4. Hubble diagram with data published in (Perlmutter et al, 1999) and corrected for deboosting. The blue dots correspond to the magnitude presented in the original publication. The pink dots correspond to the magnitude corrected for de-boosting. The solid curve presents the cosmological model with parameter $\Lambda=0$ and $\left(\Omega_{\mathrm{M}}, \Omega_{\Lambda}\right)=(2,0)$ redrawn from (Perlmutter et al, 1999).

As shown, the "de-boosting" correction of the apparent luminosities of SNIa places the effective rest-frame magnitudes below the curve corresponding to the cosmological model with parameter $\Lambda=0$ and $\left(\Omega_{\mathrm{M}}, \Omega_{\Lambda}\right)=(2,0)$.

TABLE 1

SNIa data corrected for the de-boosting effect

\begin{tabular}{|l|c|c|c|c|}
\hline \multicolumn{1}{|c|}{$\begin{array}{c}\mathrm{SN} \\
(1)\end{array}$} & $\begin{array}{c}\mathrm{z} \\
(2)\end{array}$ & $\begin{array}{c}\mathrm{m}_{\mathrm{B}}{ }^{\text {eff }} \\
(3)\end{array}$ & $\begin{array}{c}\text { Correction for } \\
\text { de-boosting } \\
(4)\end{array}$ & $\begin{array}{c}\text { Corrected } \mathrm{m}_{\mathrm{B}}{ }^{\text {corr }} \\
(5)\end{array}$ \\
\hline 1992bi & 0.458 & 23.11 & -0.82 & 22.29 \\
\hline 1994F & 0.354 & 22.38 & -0.66 & 21.72 \\
\hline 1994G & 0.425 & 22.13 & -0.77 & 21.36 \\
\hline 1994H & 0.374 & 21.72 & -0.69 & 21.03 \\
\hline 1994al & 0.42 & 22.55 & -0.76 & 21.79 \\
\hline 1994am & 0.372 & 22.26 & -0.69 & 21.57 \\
\hline 1995aq & 0.378 & 22.58 & -0.70 & 22.36 \\
\hline 1995ar & 0.453 & 23.17 & -0.81 & 22.50 \\
\hline 1995as & 0.465 & 23.33 & -0.83 & 22.83 \\
\hline 1995at & 0.655 & 23.71 & -0.88 & 21.63 \\
\hline 1995aw & 0.4 & 22.27 & -1.09 & -0.73 \\
\hline
\end{tabular}




\begin{tabular}{|c|c|c|c|c|}
\hline 1995ax & 0.615 & 23.19 & -1.04 & 22.15 \\
\hline 1995ay & 0.48 & 22.96 & -0.85 & 22.11 \\
\hline $1995 \mathrm{az}$ & 0.45 & 22.51 & -0.81 & 21.70 \\
\hline 1995ba & 0.388 & 22.65 & -0.71 & 21.94 \\
\hline $1996 \mathrm{cf}$ & 0.57 & 23.27 & -0.98 & 22.29 \\
\hline $1996 \mathrm{cg}$ & 0.49 & 23.1 & -0.87 & 22.23 \\
\hline 1996ci & 0.495 & 22.83 & -0.87 & 21.96 \\
\hline $1996 \mathrm{ck}$ & 0.656 & 23.57 & -1.10 & 22.47 \\
\hline $1996 \mathrm{cl}$ & 0.828 & 24.65 & -1.31 & 23.34 \\
\hline $1996 \mathrm{~cm}$ & 0.45 & 23.17 & -0.81 & 22.36 \\
\hline $1996 \mathrm{cn}$ & 0.43 & 23.13 & -0.78 & 22.35 \\
\hline $1997 \mathrm{~F}$ & 0.58 & 23.46 & -0.99 & 22.47 \\
\hline $1997 \mathrm{G}$ & 0.763 & 24.47 & -1.23 & 23.24 \\
\hline $1997 \mathrm{H}$ & 0.526 & 23.15 & -0.92 & 22.23 \\
\hline 1997I & 0.172 & 20.17 & -0.34 & 19.83 \\
\hline $1997 \mathrm{~J}$ & 0.619 & 23.8 & -1.05 & 22.75 \\
\hline $1997 \mathrm{~K}$ & 0.592 & 24.42 & -1.01 & 23.41 \\
\hline $1997 \mathrm{~L}$ & 0.55 & 23.51 & -0.95 & 22.56 \\
\hline $1997 \mathrm{~N}$ & 0.18 & 20.43 & -0.36 & 20.07 \\
\hline 19970 & 0.374 & 23.52 & -0.69 & 22.83 \\
\hline $1997 \mathrm{P}$ & 0.472 & 23.11 & -0.84 & 22.27 \\
\hline 1997Q & 0.43 & 22.57 & -0.78 & 21.79 \\
\hline 1997R & 0.657 & 23.83 & -1.10 & 22.73 \\
\hline $1997 \mathrm{~S}$ & 0.612 & 23.69 & -1.04 & 22.65 \\
\hline $1997 \mathrm{ac}$ & 0.32 & 21.86 & -0.60 & 21.26 \\
\hline 1997af & 0.579 & 23.48 & -0.99 & 22.49 \\
\hline 1997ai & 0.45 & 22.83 & -0.81 & 22.02 \\
\hline 1997aj & 0.581 & 23.09 & -0.99 & 22.10 \\
\hline $1997 \mathrm{am}$ & 0.416 & 22.57 & -0.76 & 21.81 \\
\hline 1997ap & 0.83 & 24.32 & -1.31 & 23.01 \\
\hline 19900 & 0.03 & 16.26 & -0.06 & 16.20 \\
\hline 1990af & 0.05 & 17.63 & -0.11 & 17.52 \\
\hline 1992P & 0.026 & 16.08 & -0.06 & 16.02 \\
\hline 1992ae & 0.075 & 18.43 & -0.16 & 18.27 \\
\hline 1992ag & 0.026 & 16.28 & -0.06 & 16.22 \\
\hline 1992al & 0.014 & 14.47 & -0.03 & 14.44 \\
\hline $1992 \mathrm{aq}$ & 0.101 & 19.16 & -0.21 & 18.95 \\
\hline $1992 b c$ & 0.02 & 15.18 & -0.04 & 15.14 \\
\hline $1992 b g$ & 0.036 & 16.66 & -0.08 & 16.58 \\
\hline 1992bh & 0.045 & 17.61 & -0.10 & 17.51 \\
\hline $1992 \mathrm{bl}$ & 0.043 & 17.19 & -0.09 & 17.10 \\
\hline 1992bo & 0.018 & 15.61 & -0.04 & 15.57 \\
\hline 1992bp & 0.079 & 18.27 & -0.17 & 18.10 \\
\hline $1992 \mathrm{br}$ & 0.088 & 19.28 & -0.18 & 19.10 \\
\hline 1992bs & 0.063 & 18.24 & -0.13 & 18.11 \\
\hline 1993B & 0.071 & 18.33 & -0.15 & 18.18 \\
\hline 19930 & 0.052 & 17.54 & -0.11 & 17.43 \\
\hline 1993ag & 0.05 & 17.69 & -0.11 & 17.58 \\
\hline
\end{tabular}


NOTES

Source of columns (1) - (3) publication (Perlmutter et al, 1999).

Col. (1) : IAU Name assigned to supernova.

Col. (2) : Geocentric redshift of supernova or host galaxy.

Col. (3) : Effective B-band peak magnitude.

Col. (4) : Correction (in magnitudes) for de-boosting.

Col. (5) : B-band peak magnitude corrected for de-boosting.

\section{Conclusions}

A Standard candle adjustment for cosmological objects with $\mathrm{z}>0.1$ appears necessary for the "de-boosting" effect, otherwise we would incorrectly assume that standard candles appear dimmer, not because of "de-boosting" but because of the vast distance, which would affect the entirety of the Standard candles ladder at cosmological distances. The "de-boosting" correction of the apparent luminosities of SNIa may further adjust and clarify cosmological models.

\section{References}

Abramowski A., Aharonian F., Ait Benkhali F., et all, 2018, Detailed spectral and morphological analysis of the shell type supernova remnant RCW 86, $\underline{\mathrm{A} \& \mathrm{~A}, 612}$

Boero E. F., Moreschi O. M., 2018, Mon. Notices Royal Astron. Soc., 475, 4683-4703

Deng Wang, Xin-He Meng. First comprehensive constraints on the Finslerian models using cosmological observations, 2018, Physics of the Dark Universe, 21, 55-60, ISSN 2212-6864

Ellis G. F. R., Maartens. R. and MacCallum. M., 2012, Relativistic Cosmology. Cambridge Univ.Press, Cambridge, pp.162, 168. https://doi.org/10.1017/CBO9781139014403

Etherington, I.M.H. (1933). On the definition of distance in general relativity, Phil. Mag. 15, 761. Reprinted as Gen. Rel. Grav. 39 (2007). https://doi.org/10.1080/14786443309462220

Granot J. \& Ramirez-Ruiz E. Jets and gamma-ray burst unification schemes, 2012, in Gammaray Bursts, eds. Chryssa Kouveliotou, Ralph A. M. J. Wijers and Stan Woosley. Published by Cambridge University Press, 2012

Kellermann, K.I., Kovalev, Y.Y., Lister, M.L. et al. Doppler boosting, superluminal motion, and the kinematics of AGN jets. 2007, Astrophys Space Sci 311, 231-239

Li K. L. et al. The X-Ray Modulation of PSR J2032+4127/MT91 213 during the Periastron Passage in 2017, 2018, ApJ 857, 123

Lister M. L. Altered luminosity functions of relativistically beamed jet populations, 2003, ApJ, $\underline{599,105-115}$

Massi M., Torricelli-Ciamponi G. Intrinsic physical properties and Doppler boosting effects in LS I +610303, 2014, A\&A 564, A23

Perlmutter, S., Aldering, G., Goldhaber G., et al. Measurements of $\Omega$ and $\Lambda$ from 42 HighRedshift Supernovae. Aph.J., 517:565-586, 1999 June 1 
Placek B. Relativistic Beaming as a Probe of Stellar and Planetary Masses. IOP Conf. Series: Journal of Physics: Conf. Series 1239 (2019) 012008, doi:10.1088/1742-6596/1239/1/012008

Shporer A., Kaplan D. L., Steinfadt J. D. R., et al. A Ground-based Measurement of the Relativistic Beaming Effect in a Detached Double White Dwarf Binary. The Astrophysical Journal Letters, Volume 725, Issue 2, pp. L200-L204 (2010).

Yang J., Brocksopp C., Corbel S., Paragi Z., Tzioumis T., Fender R. P.. A decelerating jet observed by the EVN and VLBA in the X-ray transient XTE J1752-223, 2010, Mon. Notices Royal Astron. Soc.: Letters, 409, 1, L64-L68

Zhou, J., \& Su, Y. Doppler boosting and de-boosting effects in relativistic jets of AGNs and GRBs, 2006, Proceedings of the IAU, 2(S238), 477-478

Zilberman M. "Doppler De-boosting" and the Observation of "Standard Candles" in Cosmology, Intellectual Archive, 2021, \#2552, https://doi.org/10.32370/iaj.2552 